\title{
Comparison of Competencies of Nursing Graduates of Azad Private University and State Public University
}

\author{
Reza Ghaffari ${ }^{1}$, Mojgan Mirghafourvand ${ }^{2}$, Roghayeh Abbasian $^{3 *}$ and Ali Janati ${ }^{4}$ \\ ${ }^{1} \mathrm{MD}, \mathrm{PhD}$ candidate in Medical education, Education development Center, \\ Tabriz University of Medical Sciences, Tabriz, Iran. \\ ${ }^{2}$ Assistant professor in Reproductive Health, School of nursing and midwifery, \\ Tabriz University of Medical Sciences, Tabriz, Iran. \\ ${ }^{*} \mathrm{MSc}$ in Medical Education, Education development Center, \\ Tabriz University of Medical Sciences, Tabriz, Iran. \\ ${ }^{4}$ Assocate professor in Health Services Management, School of Management \\ and Medical Informatics, Tabriz University of Medical Sciences, Tabriz, Iran.
}

DOI: http://dx.doi.org/10.13005/bbra/1945

(Received: 25 August 2015; accepted: 16 October 2015)

\begin{abstract}
Nursing competencies in the field of medical education is of particular significance and has always been under consideration by researchers of nursing education. In Medical Sciences the word capability or competency has been used as a broad concept, and basically refers to the knowledge, attitude, expertise and experience necessary for the effective implementation of a role and profession. Therefore, measuring these characteristics makes it possible to determine the ability or competency of nursing graduates. The present study aims to compare the competencies of graduates of bachelor of nursing of private and public universities of the province of Maragheh, which will be done on the bachelor of nursing graduates of the private "Azad" university and state university of Maragheh province. This study was performed as a survey from graduates of nursing undergraduate program of private and public universities in the province of Maragheh. Data collection tools were standard checklists of nursing profession of the Ministry of Health and Medical Education. The collected data were analyzed using software SPSS version 21 and the results of the evaluation of the graduates of two universities were compared with each other. At all stages, significant level of $p<0.05$ was assumed. The study found that the participants' level of knowledge and attitudes in both groups from private and public universities was low, but the status of attitudes among public university graduates with a significant $(p=0.005)$ difference was lower compared to Azad university graduates.
\end{abstract}

Key words: Competency, ability, Nursing graduates, Level of knowledge, attitude, skill.

The objectives of educational programs should generate knowledge, attitudes, and high skills in graduates in accordance with the needs of the profession and society and cause nurses have the ability to care for the patient with their professional performance. But one of the measures that can play an important role in providing necessary solutions for this crucial and necessary

\footnotetext{
* To whom all correspondence should be addressed. Tel.: +989143208695;

E-mail: abasianbonab@yahoo.com
}

task is getting feedback from graduates as key customers and the outputs of educational systems. Knowledge of the status of the graduates of universities, in order to orientate the goals of future education programs and to meet the needs of society, is considered one of the main challenges of the country. Providing suggestions and criticisms about the competence of previous nursing training programs helps in increasing the confidence and gaining clinical skills of newly graduated nurses ${ }^{1}$. The product of learning is generally considered the competency or acquired 
skill of the graduates. Competency focuses on individual abilities to perform activities related to work or life skills². In 2005, in the US the National Council of Nursing defined the competency as the ability of the individual in applying knowledge, decision-making, and psychomotor skills for playing the role of clinical nurse ${ }^{3}$. There is a general consensus on the definition of competency that competency consists of a combination of components: A) knowledge, understanding and judgment, B) a range of skills, knowledge, and psychomotor and personal techniques, and C) a range of personal characteristics and attitudes ${ }^{4}$. Professional competency has been defined as a constant and conscious use of communications, knowledge, technical skills, clinical reasoning, emotions, and values in daily practice for the benefit of the individual and society ${ }^{5}$. In Medical Sciences the word competency has been used as a broad concept and basically refers to knowledge, attitude, expertise, and necessary experiences for effective implementation of a role and profession ${ }^{6}$. Thus, by evaluating these characteristics one can measure the professional competency of nursing graduates.

Clinical competency is an important professional topic in nursing that has long been the focus of attention by officials and organizers and many efforts have been conducted to provide appropriate conditions for learning and assessing the quality of educational system in empowering graduates in this field ${ }^{7}$. Nursing is the largest group of health system and has significant potential that can affect the quality of the health care provided ${ }^{8}$. Today, all nurses, regardless of clinical duties should have technical and communication skills and possess creative and sensitive thinking in an evolutionary process. The development of these experiences is time consuming and complex ${ }^{9}$. There is a constant criticism of colleges and universities that they do not prepare students adequately and properly to begin operations in the field of nursing and this problem prevents the development of knowledge and required skills from being kept in pace with rapid changes in today's technology ${ }^{10}$. Zera'atkari et al. also in their study found that the level of learning practical skills from the viewpoint of new nursing graduates had not been complete, which this is hazardous for patients ${ }^{19}$. According to Vernon et al. (2011) the development of skills in nursing profession takes place at undergraduate level ${ }^{20}$. Evaluation of nursing graduates' ability to determine professional development and training needs is crucial and will guarantee the acquisition of knowledge and skills needed in colleges and the increase in the quality of services ${ }^{21}$. If we want our graduates to be able to solve the problems of patients, we should regularly evaluate their fundamental skills in matters related to knowledge and skills ${ }^{11}$. The study conducted in 2013 by Dadvar et al. on 56 final year nursing students showed that in skills such as applying venous catheters skill, which has been repeated and practiced during internship period of nursing students, $60 \%$ of nurses and students were evaluated as levels good, very good, and excellent and students participating in this study demonstrated better competency in skills that had higher possibility of repetition and practice ${ }^{13}$. This is in agreement with the findings of other studies ${ }^{16}$. Essentially, the opportunity for the repetition and practice of common procedures is provided with the support of instructors and colleagues in the ward. In clinical training students interacting with trainer and environment apply the learned concepts in the field of practice ${ }^{17-18}$. Professional nursing is based on performance, and measuring the achievement of the educational goals of nursing student, in the form of skill acquisition, is one of the requirements of this profession ${ }^{12}$. The aim of this study is to compare the professional competence of the graduates of bachelor of nursing of Azad and state public universities, which will be performed on the graduates of the province of Maragheh Azad and state universities.

\section{MATERIALSAND METHODS}

This study was a descriptive crosssectional study that was conducted in 2015 on 42 graduates of bachelor of nursing students of Azad private university and state university of Maragheh province. There were a total of 28 state university graduates that 21 of them participated in the study voluntarily. The number of Azad university graduates was 24, of whom 21 participated in this study voluntarily.

\section{Data collection tools included}

1. a self-assessment questionnaire containing 49 questions, including socio-demographic 
(5 questions), attitudinal (12 questions), knowledge (11 questions) and skills (21 items) characteristics. In attitudinal questions five choice scale (strongly agree, agree, somewhat agree, disagree, strongly disagree) and in knowledge and skill evaluation questions four choice scale (perfectly adequate, sufficient, low, very low) was used.

2. Multiple choice questions for evaluating the scope of the knowledge of the content of the four-year nursing theoretical courses, which included 30 questions designed by faculty members and from four main special nursing courses of: Internal Surgery Nursing (15 questions), Mental Health Nursing (5), Community Health Nursing (5 questions) and Maternal and Child Health Nursing (5 questions).

3. OSCE exam for Clinical skills evaluation including 21 select nursing clinical skills assessment standard station. These stations were prepared using nursing professional standards booklets of the Ministry of Health and Medical Education.

The validity of the self-assessment questionnaires and multiple choice questions were confirmed using content validity, such that first the self-assessment questionnaire and multiple choice questions were prepared using the continuous bachelor ofnursing program curriculum based on the latest directive from the Supreme Council of the Medical Sciences Ministry of Health and Medical Education. Then, to confirm the content validity they were sent to three faculty members of Nursing and Midwifery College of Tabriz University in Tabriz, Iran, and after obtaining their comments and making necessary modifications the final questionnaires and multiplechoice questions were prepared.

After describing different stages of the tests, the researcher gave the informed and voluntary consent forms to participants. After completing informed and voluntary consent forms the participants were divided into two groups, then the multiple-choice test was taken from the nursing graduates of the two universities and then the OSCE examination (Objective Structured Clinical Examination) was held in different stations. There were 12 OSCE stations including vital signs, oxygen therapy, lung secretions suction, nasogastric intubation, lavage, gavage, suture removal, giving medication to patients, communication skills with patients, changing dressings, venous infusions, injections, and five skills related to scrubs and observing the sterile cases, which nursing select clinical skills were evaluated in them.

The data collection tools were nursing professional standard checklists of the Ministry of Health and Medical Education. The collected data were entered into SPSS software version 21 and analyzed. The results of the graduates of two universities were compared together.

\section{RESULTS}

From the total of 52 graduates of bachelor of nursing of Azad(private) and state universities, 42 people participated in the study, of whom 42 thoroughly completed their self-assessment questionnaires and the response rate was $100 \%$. The results of the socio-demographic characteristics of the participants are shown in Table 1.

In examining the results obtained from the self-assessment questionnaire in all participants the mean and standard deviation of the score of attitudinal, knowledge, and skill scopes were evaluated to be 50.7 (6.4), 31.2 (3.6), and 70.0 (9.3) respectively (Table 2).

Also in all scopes (mean and standard deviation) the graduates of Azad university in areas of knowledge 8.31 (0.3), attitude 4.53 (5.5), and skill 4.70 (2.8) had evaluated themselves higher than state university graduates: knowledge 6.3 (1.4), attitude 9.47 (1.6), and skill 8.69 (5.10) (tables 3 and 4 ), and this difference is significant in attitudinal scope $(p=0.005)$.

According to the assessments the mean and standard deviation of the total score of knowledge resulted from four-choice exam of all participants was 15.4 (3.7). The mean and standard deviation of the score of internal surgery, mental health, community health, and maternal and child health courses are 7.8 (2.1), 2.3 (1.2), 2.3 (1.1), and 2.9 (1.0) respectively (Table 5).

Also in comparison the mean and standard deviation of the total score of knowledge in state university graduates 16.2 (3.2), was more than Azad university graduates 14.5 (4.0). In internal 
surgery course the mean and standard deviation of Azad university and state university graduates was 7.1 (1.9) and 8.5 (2.1) respectively, which this shows a significant difference $(p=0.042)$ in internal surgery course (Table 6).

In mental health, community health, and mother and child health course scores Azad university results were: mental health: 2.2 (1.2), community health 2.4 (1.0), and mother and child health 2.8 (1.1) and state university results were: mental health: 2.4 (1.3), community health 2.1 (1.1), and mother and child health 3.1 (1.0), which in comparing the two groups no significant difference was observed in the mean of the scores of these courses (Table 6).

The status of the self-assessment of knowledge, attitude, and skill in state university and Azad university were compared with each other, which the results are shown in table 7.

After evaluating the results of the OSCE exam in general, from 21 practical skills listed in table 8, participants received a mean score of good in 19 skills. In the skill of removing sutures the mean score was at medium level and in the skill of adding solution to container in sterile environment had a mean score of weak. In total, the participants

Table 1. Demographic characteristics of participants

\begin{tabular}{|c|c|c|c|c|}
\hline & & $\begin{array}{l}\text { Total participants }(\mathrm{n}=42) \\
\text { Numbers (Percent) }\end{array}$ & $\begin{array}{l}\text { State university } \\
\qquad(\mathrm{n}=21)\end{array}$ & $\begin{array}{l}\text { Azad university } \\
\qquad(\mathrm{n}=21)\end{array}$ \\
\hline \multicolumn{2}{|c|}{ Mean age (Standard deviation) } & $23.0(1.4)$ & $23.2(1.2)$ & $22.9(1.2)$ \\
\hline \multirow[t]{2}{*}{ Sex } & Female & $33(78.6)$ & $12(57.1)$ & $21(100)$ \\
\hline & Male & $9(21.4)$ & 9 (42.9) & 0 \\
\hline \multirow[t]{2}{*}{ Marital status } & Single & $30(71.4)$ & $16(76.2)$ & $14(66.7)$ \\
\hline & Married & $12(28.6)$ & $5(23.8)$ & 7 (33.3) \\
\hline \multirow[t]{2}{*}{ Occupation } & Employed & $3(7.1)$ & $2(9.5)$ & $1(4.8)$ \\
\hline & Unemployed & 39 (92.9) & $19(90.5)$ & $20(95.2)$ \\
\hline \multirow[t]{2}{*}{ Locality status } & Local & $27(64.3)$ & $6(28.6)$ & $21(100)$ \\
\hline & Non-local & $15(35.7)$ & $15(71.4)$ & 0 \\
\hline
\end{tabular}

Table 2. The status of self-assessment of knowledge, attitude, and skill in all of participants

\begin{tabular}{lccc}
\hline Variable & Mean (standard deviation) & Achieved score range & Achievable score range \\
\hline Knowledge & $31.2(3.6)$ & $22-38$ & Nov-44 \\
Attitude & $50.7(6.4)$ & $37-59$ & Dec-60 \\
Skill & $70.0(9.3)$ & $42-84$ & $21-84$ \\
\hline
\end{tabular}

Table 3. The status of self-assessment of knowledge, attitude, and skill in Azad university of Maragheh

\begin{tabular}{lccc}
\hline Variable & Mean (standard deviation) & Achieved score range & Achievable score range \\
\hline Knowledge & $31.8(3.0)$ & $27-38$ & Nov-44 \\
Attitude & $53.4(5.5)$ & $40-59$ & Dec-60 \\
Skill & $70.4(8.2)$ & $54-84$ & $21-84$ \\
\hline
\end{tabular}

Table 4. The status of self-assessment of knowledge, attitude, and skill in the state university of Maragheh

\begin{tabular}{lccc}
\hline Variable & Mean (standard deviation) & Achieved score range & Achievable score range \\
\hline Knowledge & $30.6(4.1)$ & $22-37$ & Nov-44 \\
Attitude & $47.9(6.1)$ & $37-59$ & Dec-60 \\
Skill & $69.8(10.5)$ & $42-84$ & $21-84$ \\
\hline
\end{tabular}


in no skill received a mean score of excellent (table 8).

In comparing the status of the results of OSCE exam of the graduates of the two groups, Azad university had higher scores than state university in ten skills mentioned in the following with related means and standard deviations: communications skills 1.8 (0.2), measuring pulse $1.9(0.4)$, Measuring breathing 1.9 (0.3), measuring temperature $1.9(0.2)$, catheter insertion $1.6(0.2)$, inserting NG tube $1.7(0.2)$, lavage $1.7(0.3)$, gavage $1.6(0.1)$, giving medication

Table 5. Status / level of evaluation knowledge of all participants

\begin{tabular}{lccc}
\hline Variable & Mean (standard deviation) & Achieved score range & Achievable score range \\
\hline Knowledge Total Score & $15.4(3.7)$ & 22-Jun & $0-30$ \\
Internal Surgery & $7.8(2.1)$ & $12-$ Apr & $0-15$ \\
Mental Health & $2.3(1.2)$ & $0-5$ & $0-5$ \\
Community Health & $2.3(1.1)$ & $0-4$ & $0-5$ \\
Mothers and Babies & $2.9(1.0)$ & 5-Jan & $0-5$ \\
\end{tabular}

Table 6. Comparison of the status of the assessment of knowledge between state university and Azad University graduates in the city of Maragheh

\begin{tabular}{lccc}
\hline Variable & Azad university & State university & $\mathrm{P}$ \\
\hline Knowledge total score & $14.5(4.0)$ & $16.2(3.2)$ & 0.15 \\
Internal surgery & $7.1(1.9)$ & $8.5(2.1)$ & 0.042 \\
Mental health & $2.2(1.2)$ & $2.4(1.3)$ & 0.718 \\
Community health & $2.4(1.0)$ & $2.1(1.1)$ & 0.483 \\
Mothers and babies & $2.8(1.1)$ & $3.1(1.0)$ & 0.366 \\
\hline
\end{tabular}

Table 7. Comparison of the status of self-assessment of knowledge, attitude, and skill in state university and Azad university graduates

\begin{tabular}{lccc}
\hline Variable & Azad university & State university & P \\
\hline Knowledge & $31.8(3.0)$ & $30.6(4.1)$ & 0.317 \\
Attitude & $53.4(5.5)$ & $47.9(6.1)$ & 0.005 \\
Skill & $7.4(8.2)$ & $69.8(10.5)$ & 0.859 \\
\hline
\end{tabular}

to patient $1.8(0.1)$, and adding solution to container in sterile environment $0.9(0.4)$, which in five skills including the following skills: inserting nasogastric tube $(p=0.000)$, lavage $(p=0.003)$, gavage $(0.003)$, giving medication to patients $(\mathrm{p}=0.005)$, and adding solution to container in sterile environment $(p=0.000)$ had higher mean score with a significant difference with respect to the graduates of state university (Table 9).

The graduates of state university had also higher scores compared with Azad university in the following nine skills with score mean and standards deviations of: injection 1.5 (0.2), preparing serum $1.7(0.2)$, oxygen therapy $1.7(0.2)$, suction of secretions $1.7(0.3)$, change of dressing
1.3 (0.3), opening and closing a sterile package 1.7 (0.4), hand washing 1.8 (0.2), wearing gown 2.0 (0.0), and wearing gloves, which in three skills including suction of lung secretions ( $p=0.025)$, opening and closing a sterile package $(p=0.000)$, and wearing gown $(\mathrm{p}=0.001)$ skills with a significant difference had a higher mean score compared to Azad university graduates (table 9). In skills of measuring blood pressure and suture removal both groups were similar (table 9).

Comparison of clinical skills of two groups of nursing graduates from Azad and state universities of the city of Maragheh showed that despite the similarity of the clinical education programs of two studied groups, implementation 
Table 8. Status of OSCE (clinical skills) in all participants

\begin{tabular}{lccc}
\hline Variable & $\begin{array}{c}\text { Mean (standard } \\
\text { deviation) }\end{array}$ & $\begin{array}{c}\text { Achieved score } \\
\text { range }\end{array}$ & $\begin{array}{c}\text { Achievable score } \\
\text { Range }\end{array}$ \\
\hline communication skills & $1.8(0.2)$ & $1.5-2.0$ & $0-2$ \\
Measuring pulse & $1.8(0.4)$ & $0.5-2.0$ & $0-2$ \\
Measuring respiration & $1.9(0.3)$ & $1.0-2.0$ & $0-2$ \\
Blood pressure measurement & $1.9(0.4)$ & $1.5-2.0$ & $0-2$ \\
Temperature measurement & $1.9(0.2)$ & $1.1-2.0$ & $0-2$ \\
Injections & $1.5(0.2)$ & $1.4-1.9$ & $0-2$ \\
Catheter insertion & $1.5(0.2)$ & $1.2-2.0$ & $0-2$ \\
Preparing serum & $1.7(0.2)$ & $1.1-2.0$ & $0-2$ \\
Oxygen therapy & $1.6(0.2)$ & $1.0-2.0$ & $0-2$ \\
Suction & $1.7(0.2)$ & $1.1-2.0$ & $0-2$ \\
Dressing change & $1.3(0.2)$ & $0.5-1.9$ & $0-2$ \\
Removing sutures & $0.9(0.2)$ & $0.5-1.9$ & $0-2$ \\
NG tube insertion & $1.5(0.2)$ & $1.1-2.0$ & $0-2$ \\
Lavage & $1.5(0.3)$ & $0.7-2.0$ & $0-2$ \\
Gavage & $1.4(0.2)$ & $1.0-1.8$ & $0-2$ \\
Giving medicine to patients & $1.7(0.1)$ & $1.3-1.9$ & $0-2$ \\
Opening and closing sterile packages & $1.3(0.5)$ & $0.7-2.0$ & $0-2$ \\
Adding the solution to the container in & & & \\
a sterile environment & $0.5(0.5)$ & $0.0-1.5$ & $0-2$ \\
Hand washing & $1.7(0.3)$ & $0.7-2.0$ & $0-2$ \\
Wearing gown & $1.9(0.2)$ & $1.2-2.0$ & $0-2$ \\
Wearing gloves & $1.8(0.2)$ & $1.3-2.0$ & $0-2$ \\
\hline
\end{tabular}

Table 9. Comparison of OSCE (clinical skills) between Maragheh state and Azad universities

\begin{tabular}{lccc}
\hline Variable & Azad university & State university & P \\
\hline communication skills & $1.8(0.2)$ & $1.8(0.2)$ & 0.756 \\
Measuring pulse & $1.9(0.4)$ & $1.8(0.5)$ & 0.427 \\
Measuring respiration & $1.9(0.3)$ & $1.8(0.2)$ & 0.669 \\
Blood pressure measurement & $1.9(0.1)$ & $1.9(0.2)$ & 0.427 \\
Temperature measurement & $1.9(0.2)$ & $1.8(0.2)$ & 0.496 \\
Injections & $1(0.2) .4$ & $1.5(0.2)$ & 0.416 \\
Catheter insertion & $1.6(0.2)$ & $1.5(0.1)$ & 0.728 \\
Preparing serum & $1.6(0.2)$ & $1.7(0.2)$ & 0.705 \\
Oxygen therapy & $1.6(0.3)$ & $1.7(0.2)$ & 0.062 \\
Suction & $1.6(0.2)$ & $1.7(0.3)$ & 0.025 \\
Dressing change & $1.2(0.2)$ & $1.3(0.3)$ & 0.614 \\
Removing sutures & $0.9(0.2)$ & $0.9(0.3)$ & 0.469 \\
NG tube insertion & $1.7(0.2)$ & $1.4(0.2)$ & 0 \\
Lavage & $1.7(0.3)$ & $1.3(0.3)$ & 0.003 \\
Gavage & $1.6(0.1)$ & $1.3(0.2)$ & 0 \\
Giving medicine to patients & $1.8(0.1)$ & $1.7(0.1)$ & 0.005 \\
Opening and closing sterile packages & $1.0(0.2)$ & $1.7(0.4)$ & 0 \\
Adding the solution to the container in a & & & \\
sterile environment & $0.9(0.4)$ & $0.0(0.1)$ & 0 \\
Hand washing & $1.7(0.3)$ & $1.8(0.2)$ & 0.47 \\
Wearing gown & $1.8(0.2)$ & $2.0(0.0)$ & 0.001 \\
Wearing gloves & $1.8(0.2)$ & $1.9(0.2)$ & 0.217 \\
\hline
\end{tabular}


of the internship programs for students of both universities in clinical fields at the same hospitals, and commonality of most clinical instructors of both groups, there are significant differences in most practical skills between the two groups.

Azad university graduates had higher mean scores in five skills compared to graduates of state university with significant differences, the skills included: inserting NG tube ( $\mathrm{p}=0.000)$, lavage $(p=0.003)$, gavage $(p=0.000)$, giving medication to patients $(\mathrm{p}=0.005)$, and adding solution to a container in sterile environment $(\mathrm{p}=0.000)$, and state university graduates also had higher mean scores compared with Azad university graduates in three skills of lung secretions suction ( $p=0.025)$, opening and closing a sterile package $(\mathrm{p}=0.000)$, and wearing gown $(\mathrm{p}=0.001)$.

The low mean score of knowledge in the graduates of both groups is an indication of a gap in knowledge between the desirable and available status; mean scores are (16.2 out of 30) and (14.5 out of 30). Although one reason for this drop could be the gap between the time of taking theoretical courses and time of graduation, they should not addressing and favoring the development of the level of clinical skills.

\section{DISCUSSION}

Reaching professional standards in medical sciences including in nursing profession takes place through achieving the clinical skill goals of the profession ${ }^{13}$. Nursing graduates should have basic clinical skills. Nursing students must have the ability to provide services in this area after graduation ${ }^{13}$. In this study, we evaluated the professional competence of nursing graduates of Azad and state universities of Maragheh in 2015 and before entering work environment and compared them with each other and the status of the knowledge, attitude, and skill of graduates were studied and measured. Also important procedures such as measuring vital signs, injections, catheter insertion, wound dressing, nasogastric intubation, suctioning lung secretions, lavage, gavage, observing sterile matters, etc. were evaluated that graduates in neither group managed to obtain excellent score in performing theses skills. In comparing the two groups a significant difference in a number of skills was observed. In examining the results of the self-assessment questionnaire in the attitude of all participants mean and standard deviation of attitudinal 7.50 (4.6), knowledge 2.31 (6.3), and skill 0.70 (3.9) scopes were evaluated (table 2). This mean that is indicative of poor attitude of the participants confirms the results of other studies that showed nursing graduates possessed low motivation, interest, and commitment ${ }^{14}$. The results also showed a serious weakness in the level of awareness and knowledge of participants, which both the self-assessment of the participants 2.31 (6.3) and the results obtained from the multiplechoice test 4.15 (7.3) confirm it. The reason for the decline in the skill level of graduates differs. Researchers believe that a lack of motivation, interest and commitment among students is one of the causes of the decline in learning and quality of clinical education. A study in 2002 indicated that colleges do not prepare students desirably for clinical performance. And there are deficiencies in teaching clinical skills to students ${ }^{14}$. In national studies also similar results regarding deficiencies in nursing curriculum in supplying nursing students with the necessary level of knowledge and skills are seen ${ }^{15}$. The study found that the level of knowledge and attitude of participants of both groups (Azad and state universities graduates) is low, but in comparison the status of attitude among the graduates of state university was lower than that of the graduates of Azad university with a significant difference $(p=0.005)$. It was also determined that the mean score of the internal surgery course among Azad university graduates was lower than that of the state university graduates with a significant difference $(\mathrm{p}=0.042)$. Accountability of universities in proper implementation of educational curriculums and training capable graduates with regard to professional competencies and existence of various views with regard to the difference in capabilities of the graduated students from private and public universities is one of the reasons for doing this research. The obtained results show that despite using potentials, possibilities, and facilities of clinical education and the use of common faculty members in the region by both universities there are still significant differences regarding the competency of the graduates of the two Azad and state universities of the city of 
Maragheh. One of the limitations of this study was the small number of graduates of Azad and state universities of Maragheh in 1394, and due to the small number of graduates in $1394{ }^{52}$, the number of students participating in the study was also $\operatorname{low}^{42}$. It is recommended that similar research to be done in this field in other cities and universities, in order for the results to have higher credibility in terms of generalization of the results.

\section{ACKNOWLEDGMENTS}

With regard to performing this research, we would like to acknowledge and thank the managers of nursing groups, educational deputies, and research deputies of Azad and state Universities of Maragheh who supported us sincerely in implementing different stages of this examination.

\section{REFERENCES}

1. Toulabi T, Alhani F.A comparative study of educational programs' objectives for professional needs from the viewpoints of nursing graduates in three periods of three years. Yafteh. 2011; 12(2) :0-0

2. Adam S. A consideration of the nature, role, application and implications for European education of employing 'learning outcomes' at the local, national and international levels. University of Westminster. 2004.

3. Hsieh SI, Hsu LL. An outcome-based evaluation of nursing competency of baccalaureate senior nursing students in Taiwan.Nurse education today. 2013; 33(12):1536-45.

4. Lofmark, Anna et al, Competence of newly graduated nurses-a comparison of the perceptions of qualified nurses and students, Journal of Advanced Nursing. 2006; 53: 721-728.

5. Epstein, Ronald M. Hundert, Edward $\mathrm{M}_{\mathrm{i}}$ Defining and assessing professional competence, JAMA 2002; 287: 226-235.

6. Mitchell ML, et al. The objective structured clinical examination (OSCE): Optimizing its value in the undergraduate nursing curriculum. Nurse Education Today 2009; 29(4): 398-404.

7. Farnia F, et al, Nursing graduates employment at the beginning of their clinical skills assessment, Journal of Medical Sciences, 1384; 5(14).

8. Reily DE. Behavioral objectives evaluation in nursing .2nd ed. New York: Appleton Century
Crofts Co, 1980; 89.

9. Delbueno JD. Experience, education, and nurses' ability to make clinical judgment. Nurs- Health Care 1990; 11: 290.94

10. Evenson WM. The consistency of clinical nursing education with employer expectation, perception of registered nurses in Montana. EDC Dissertation, university of Montana. 1989; 11-12

11. AlvesDelima A, et al. Mini-clinical Evaluation Exercise (Mini-CEX). Global Standard in Medical Education for Better Health Care. 2003; 32.

12. Raines D. Nursing practice competency of accelerated bachelor of science in nursing program students. J Prof Nurs 2010; 26:162167.

13. Dadvar Leila, et al., Evaluation of Educational Goals Achievement in Fundamental Nursing Clinical Skills: Application OSCE among Senior Nursing Students in ICU, Journal of Knowledge \& Health, 2014; 2015; 9(4).

14. Rolfe IE, Sanson Fisher R W. Translating learning principles into practice: a new strategy for learning clinical skills. Med Educ 2002; 36(4): 345-352.

15. Dadgari A, Dadvar L, Rezaee M, Mirzaee M, Nazari P, Naseri L. Nurses' educational needs on drug dosage calculation. Sobhe Daneshgah 2009; 12: 26.[Persian].

16. Engum SA, Jeffries P, Fisher L. Intravenous catheter training system: Computer-based education versus traditional learning methods. Am J Surg 2003; 186(1): 67-74.

17. Alves Delima A, Henquin A, Thierer J, Lamri S, Paulin J, Belcadtro F. Mini-clinical Evaluation Exercise (Mini-CEX). Global Standard in Medical Education for Better Health Care. 2003; 32.

18. Mackay SJ, Anderson AC, Hogg P. Preparedness for clinical practice-perceptions of graduates and their work supervisors. Radiography 2008; 14(3):226-232.

19. Zeraatkari K. Learning clinical skill: newly graduates nurse students. Abstract of $3^{\text {th }}$ National Conference on Medical Education 1998; 168. [Persian].

20. Vernon RCMPE. Confidence in competence: legislation and nursing in New Zealand. International Nursing Review 2011;58:103-108.

21. Marshburn DMEMKSMS. Relationships of new nurses perceptions and measured performance-based clinical competence. Continuing Education in Nursing 2009; 40(9): 426-432. 